\title{
Quantitative assessment of blood-brain barrier permeability in multiple sclerosis using 68-Ga-EDTA and positron emission tomography
}

\author{
C POZZILLI,* S BERNARDI, ${ }^{*}$ L MANSI, $\dagger$ P PICOZZI $\ddagger$ F IANNOTTI, \\ B ALFANO,$\dagger$ L BOZZAO, $*$ GL LENZI, ${ }^{*}$ M SALVATORE, $\dagger$ P CONFORTI,+ \\ C FIESCHI*
}

From the Department of Neurological Sciences, ${ }^{*}$ University of Rome "La Sapienza", Institute of Neurosurgery, $\ddagger$ University of Naples, National Cancer Institute "Fondazione Sen. G. Pascale", $\dagger$ Naples, Italy.

SUMMARY Fifteen patients with definite multiple sclerosis were examined with high volume delayed (HVD) CT scan and positron emission tomography (PET) using 68-Ga-EDTA as a tracer. The passage of $68-$ Ga-EDTA across the blood-brain barrier (BBB) was measured by using multiple graphical analysis. This method permits the simultaneous calculation of a blood to brain influx constant $\mathrm{Ki}\left(\mathrm{ml} / \mathrm{g}^{-1} \mathrm{~min}^{-1}\right)$ and of the plasma volume $\mathrm{Vp}\left(\mathrm{ml} / \mathrm{g}^{-1}\right)$. Focal areas of abnormal CT enhancement and pathological accumulation of 68-Ga-EDTA were visualised in four patients who were all examined during a clinical exacerbation of the disease. The mean $\mathrm{Ki}$ value measured in these areas was $12 \cdot 5$, SD 3.4 indicating a moderate but significant increase of BBB permeability compared with the value found in normal tissue $(3 \cdot 2, \operatorname{SD} 0 \cdot 9)$. No parallel increase in Vp values was found in these pathological areas. Quantitative data obtained with PET seems to provide further insight into the study of $\mathrm{BBB}$ function in multiple sclerosis.

In postmortem perfusional studies utilising trypan blue, Broman first demonstrated a disturbance of blood-brain barrier (BBB) permeability in some multiple sclerosis plaques. ${ }^{1}$

Subsequently, using CT scan after the intravenous injection of a contrast medium, regions of abnormal enhancement, reflecting extravasation of iodine through a damaged BBB, have frequently been reported in patients with multiple sclerosis. ${ }^{2-4}$ The detection of these enhancing areas was found to be markedly increased when the patients were studied during recent clinical exacerbation ${ }^{56}$ and when a high volume delayed CT scanning technique (HVD-CT) was employed. ${ }^{78}$ However, enhanced CT shows only a BBB alteration without being able to quantify the alteration itself.

The measurement of BBB permeability in humans has recently been obtained using positron emission tomography (PET). ${ }^{9-13}$ In a previous paper ${ }^{14}$ we

\footnotetext{
Address for reprint requests: Carlo Pozzilli, Dipartimento di Scienze Neurologiche, Università "La Sapienza" Viale dell 'Università, 30 00185 Roma, Italy.
}

Received 10 July 1987 and in revised form 31 December 1987. Accepted 5 January 1988 described a simple and non invasive method for quantifying BBB permeability using 68-Ga-EDTA and PET. The technique was found to be sensitive and accurate for the measurement of BBB disruption associated with brain tumours.

The purpose of the present investigation was to elucidate the value of 68-Ga-EDTA and PET in the study of BBB abnormalities associated with multiple sclerosis.

\section{Material and methods}

Patients A total of 15 patients with multiple sclerosis, three men and 12 women, aged 17 to 51 years (mean $30 \mathrm{yr}$ ) and four age-matched control subjects were selected for the study. The diagnosis of definite multiple sclerosis was determined by the clinical criteria of Schumacher et al. ${ }^{15} \mathrm{CSF}$ studies and evoked potentials were available in all patients. Magnetic resonance imaging was performed on five patients.

Eight patients experienced a clinical exacerbation at the time of the study; two were in remission and five had a chronic progressive form of multiple sclerosis. All patients had clinical or CT scan suggestion of at least one supratentorial area of the demyelinating process, although in the five patients with the chronic progressive form of the disease. the clinical decline was evidenced mainly by neurological 
Table 1 HVD-CT and PET scan findings in 15 patients with definite multiple sclerosis

\begin{tabular}{lcccc}
\hline \multirow{2}{*}{$\begin{array}{l}\text { Disease's } \\
\text { activity }\end{array}$} & \multicolumn{2}{l}{ HVD-CD scan } & & $\begin{array}{l}\text { PET scan } \\
\text { focal abnormal } \\
\text { 68-Ga-EDTA-uptake }\end{array}$ \\
\cline { 2 - 5 } & Low density areas & Large CSF & Enhancing areas & 4 \\
\hline $\begin{array}{l}\text { Exacerbation }(\mathrm{n}=8) \\
\text { Remission }(\mathrm{n}=2)\end{array}$ & 7 & 4 & 4 & 0 \\
Chronic progression $(\mathrm{n}=5)$ & 1 & 1 & 0 & 0 \\
Total $(\mathrm{n}=15)$ & 12 & 5 & 0 & 4 \\
\hline
\end{tabular}

signs and symptoms related to a spinal cord involvement. Patients had no steroid or other pharmaceutical treatment at the time of the study; in those with acute relapses, the beginning of therapy was postponed until the completion of the HVD-CT and PET study. Consent to participate in the study was signed by all patients and volunteers prior to initiation. HVD CT scan After a non contrast CT scan, routine enhancement (40 $\mathrm{gm}$ of iodine) was administered and a CT scan was performed. When the scan terminated, a second dose of contrast medium ( $40 \mathrm{gm}$ of iodine) was administered and a delayed (45-60 minutes) scan was also obtained.

PET scan The complete procedure used for studying BBB permeability with 68-Ga-EDTA and PET has been described elsewhere. ${ }^{14}$ After a transmission scan to allow for attenuation correction, $6-8 \mathrm{mCi}$ of $68-\mathrm{Ga}-\mathrm{EDTA}$ were injected intravenously. Then 16 scans, 300 seconds each, were performed on a single ring Neuro-ECAT scanner (CTI) with a spatial resolution of $8.7,1.0 \mathrm{~mm}$ (mean, SD) full width at half maximum (FWHM) in a slice of $16 \mathrm{MM}$. The tomographic level of the slice examined was selected on the basis of CT enhancement, when present. Otherwise, a standard slice through the body of the lateral ventricles, approximately at $\mathrm{OM}+6 \mathrm{~cm}$ was chosen.

The measurement of 68-Ga-EDTA passage across BBB was achieved by multiple time graphical analysis. ${ }^{16-18}$ This method allows for the simultaneous calculation of a blood to brain influx constant $\mathrm{Ki}\left(\mathrm{ml} \mathrm{g}^{-1} \mathrm{~min}^{-1}\right)$ and of the plasma volume $\mathrm{Vp}\left(\mathrm{ml} \mathrm{g}^{-1}\right)$. The tracer time activity curve in the blood, corresponding to the input function, was calculated by a non-invasive method that avoids arterial sampling. This method utilises a region of interest (ROI) drawn on the sagittal sinus and one venous blood sample. The time activity curve derived from the sinus image was converted in absolute terms by measuring the actual activity of the syringe blood withdrawn at a fixed time. The values measured with this procedure are comparable to those derived using standard arterial sampling. ${ }^{14}$

The spatial activity distribution in the scanned plane was reconstructed using a filtered back projection with the ramp filter and an attenuation correction measured by transmission scan. After reconstruction, parametric images of $\mathbf{K i}$ and $\mathrm{Vp}$ were obtained utilising the entire 16 scans dynamic sequence.

Small ROIs containing a minimum of 30 pixels were placed on the areas showing an abnormal 68-Ga-EDTA uptake in the parametric images. The values obtained from those ROIs were plotted in a graphic form according to the multiple time graphical analysis method, and used to calculate $\mathrm{Ki}$ and $\mathrm{Vp}$. The pattern of the plotted data was linear in all the measurements thus indicating the progressing accumulation of the tracer in the brain during the entire scanning period. The $\mathrm{Ki}$ values of these regions were considered of pathological significance when exceeding the 2SD of the mean of the normal values.

Additionally in all patients and in normal subjects, four standard ROIs were placed on the frontal and parietal lobes and averaged in order to obtain a whole-brain $\mathrm{Ki}$ and $\mathrm{Vp}$ value for each subject.

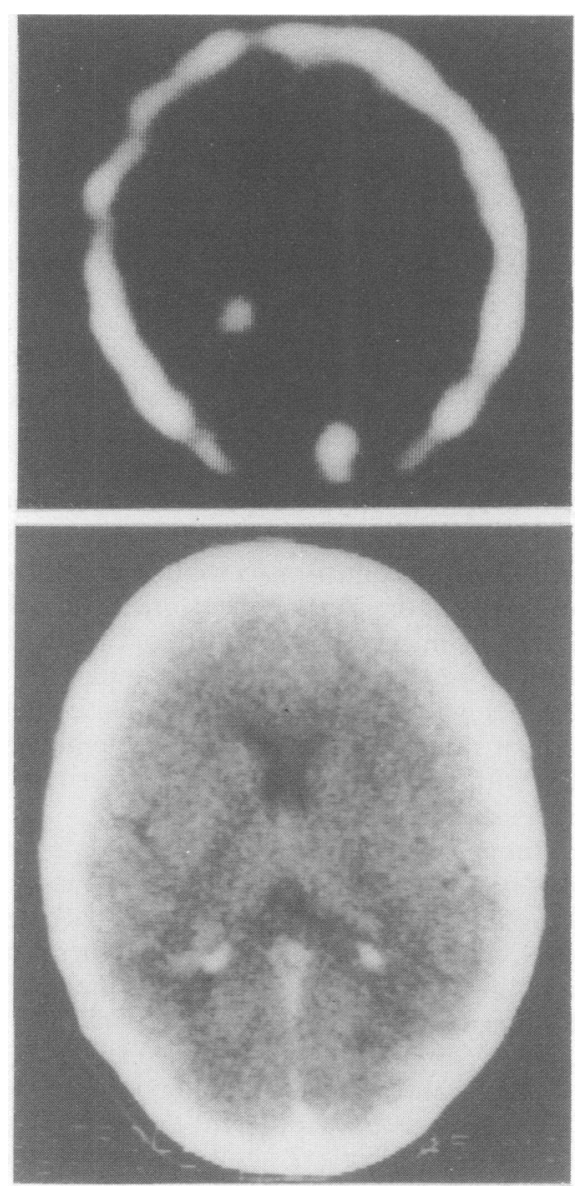

Fig 1 PET and HVD-CT scans of a patient with multiple sclerosis examined during an exacerbation of the disease. Note the focal increase of 68-Ga-EDTA uptake corresponding to the left periventricular CT enhanced area. 


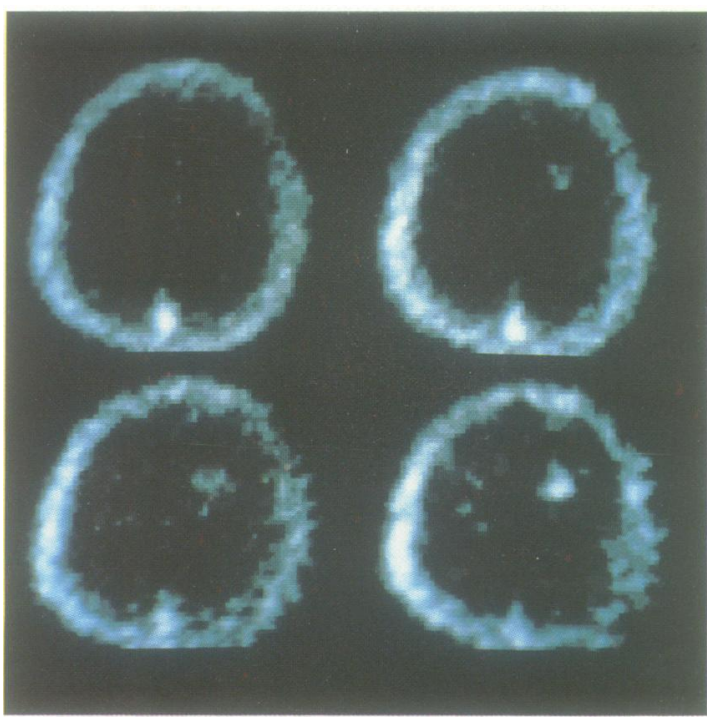

Fig 2 Sequential scans obtained at 20, 40, 60, 80 minutes after an intravenous injection of 68-Ga-EDTA. Focal areas of increased tracer uptake are shown both on the right (early scans) and on the left (tardive scan) hemisphere.

\section{Results}

Table 1 shows CT and PET scans abnormalities in multiple sclerosis patients divided into three subgroups according to the activity of the disease.

Focal areas of pathological accumulation of 68-Ga-EDTA were visualised in four patients, all examined during a clinical exacerbation of the disease. The location of the pathological areas was clearly related to the clinical relapse in three of these cases. In one patient, presenting with left hemiparesis, PET scan showed evidence of bilateral lesions. Extent and location of abnormal PET areas roughly corresponded with the enhanced areas seen on HVD-CT images, as shown in fig 1 .

A dynamic PET sequence showing multiple areas of increased 68-Ga-EDTA uptake is reported in fig 2 . In this patient, an early 68-Ga-EDTA pathological uptake is clearly detected on the right hemisphere, whereas multiple pathological areas on the left hemisphere appear only in the delayed scans. $\mathrm{Ki}$ value measured in the area of right hemisphere was three times higher than the corresponding value in the left hemisphere, suggesting a different degree of regional BBB impairment. Table 2 shows mean $\mathrm{Ki}$ and $\mathrm{Vp}$ values in brain areas of multiple sclerosis patients, showing a significant increase of BBB permeability. The increased $\mathrm{Ki}$ value in these areas was not accompanied by a parallel increase of the vascular space $(\mathrm{Vp})$, which was within the normal range.
Table 2 Mean Ki and Vp values in "abnormal" ROIs of multiple sclerosis patients (a) and in "normal" ROIs of control subjects (b)

\begin{tabular}{|c|c|c|}
\hline & $\begin{array}{l}\mathrm{Ki} \times 10^{-4} \\
\left(\mathrm{ml}^{-1} \mathrm{~g}^{-1} \mathrm{~min}^{-1}\right)\end{array}$ & $\begin{array}{l}V p \\
\left(m l . g^{-1}\right)\end{array}$ \\
\hline \multirow{2}{*}{$\begin{array}{l}\text { (a) ROIs with increased B-B-B } \\
\text { permeability }(\mathrm{n}=7) \\
\text { (b) ROIs with normal B-B-B } \\
\text { permeability }(\mathrm{n}=4)\end{array}$} & $12 \cdot 5, \mathrm{SD} 3 \cdot 4^{*}$ & $3 \cdot 3$, SD 0.3 \\
\hline & 3.2 , SD 0.9 & 3.7, SD 0.7 \\
\hline
\end{tabular}

$* \mathrm{p}<0.001(t$ test $)$

Whole brain $\mathrm{Ki}$ and $\mathrm{Vp}$ values of multiple sclerosis patients and four normal subjects are shown in table 3. No significant difference was found for either the parameters between normal subjects or each of the multiple sclerosis subgroups.

\section{Discussion}

PET has been employed to assess BBB integrity in patients with cerebral infarction, brain tumours and dementia. ${ }^{19-23}$ Recently, Brooks et al ${ }^{9}$ found normal BBB permeability in multiple sclerosis. However, their patients were in remission at the time of the study.

As frequently reported ${ }^{56}$ we also have observed a correlation between BBB alteration and clinical activity of multiple sclerosis. Both HVD-CT and PET studies clearly showed areas of BBB abnormalities in four of the eight patients examined during "acute exacerbation" before the initiation of antiinflammatory treatment. The negative findings in the remaining four patients could be explained by the fact that clinical exacerbations were associated with new symptoms reflecting an involvement of brain stem in two cases, and optic nerve and spinal cord in one case each. The absence of a pathological BBB increased permeability is justified in two patients examined during clinical remission and in five patients with chronic progressive multiple sclerosis whose clinical decline was shown mainly by signs and symptoms related to spinal cord involvement.

In the present series enhancing areas demonstrated by the HVD-CT scan were also detected by PET. However, a comparison of these two techniques was not the aim of this study and it is further limited at the present time since a single ring positron camera was

Table 3 Whole brain Ki and Vp values in 15 multiple sclerosis patients and in 4 normal subjects

\begin{tabular}{|c|c|c|}
\hline $\begin{array}{l}\text { Disease's } \\
\text { activity }\end{array}$ & $\begin{array}{l}\mathrm{Ki} \times 10^{-4} \\
\left(\mathrm{ml} \cdot \mathrm{g}^{-1} / \mathrm{min}^{-1}\right)\end{array}$ & $\begin{array}{l}V p \\
\left(m l \cdot g^{-1}\right)\end{array}$ \\
\hline $\begin{array}{l}\text { Exacerbation }(n=8) \\
\text { Remission }(n=2) \\
\text { Chronic progression }(n=5)\end{array}$ & $\begin{array}{l}3 \cdot 6 \text {, SD } 1 \cdot 1 \\
3 \cdot 3 \text {, SD } 0 \cdot 3 \\
3 \cdot 1, \text { SD } 0 \cdot 9\end{array}$ & $\begin{array}{l}4 \cdot 0, \text { SD } 0 \cdot 6 \\
4 \cdot 5, \text { SD } 0 \cdot 1 \\
4 \cdot 1, \text { SD } 0 \cdot 8\end{array}$ \\
\hline Normal controls $(n=4)$ & 3.0, SD 0.7 & $3 \cdot 5$, SD 0.7 \\
\hline
\end{tabular}


used. Therefore the dynamic images of 68-Ga-EDTA accumulation were obtained only in the single PET slice chosen in advance on the basis of CT findings. This problem may be overcome by employing PET systems equipped to image multiple slices simultaneously.

The major advantage of PET is to add quantitative information on BBB permeability to the qualitative morphological information obtained by HVD-CT scan. However, the measurement of BBB permeability, expressed in terms of transfer constant (Ki), may be affected by partial volume effect. Therefore, if the lesion with increased BBB permeability is smaller with respect to the ROI sampled, then a very and perhaps minute increase in Ga-EDTA uptake would be measured despite a marked local elevation in $\mathrm{Ki}$. Although we chose relatively small ROIs (not less than $1.65 \mathrm{~cm}^{2}$ ), the possibility of an underestimation of $\mathrm{Ki}$ values cannot be completely ruled out owing to the frequently smaller size of multiple sclerosis plaques.

The utilisation of a "non-invasive" approach to calculate the input function may represent another possible source of error in absolute measurements of $\mathrm{Ki}$ and $\mathrm{Vp}$ values. The "non-invasive" method was designed to obviate the need to measure an arterial plasma input function and has been validated elsewhere. ${ }^{14} \mathrm{~A}$ possible source of error is the difference between the very first part of the input curves obtained from the arterial blood and from the sinus image. This results mainly in a difference in the plot of the first point. However, although the initial parts of the two curves do not overlap until 15 minutes, ${ }^{14}$ it should be noted that the calculation of $\mathrm{Ki}$ and $\mathrm{Vp}$ is based on straight line least-square fitting of 16 points (scans) and, as a rule, the weight of the first scan is negligible.

Despite these limitations PET quantitative data seem to provide further insight into the functional state of BBB in multiple sclerosis. BBB disruption, when present, is strictly focal and of a moderate entity. The mean $\mathrm{Ki}$ values, measured in the areas with an evident increase of BBB permeability, were 12.5 , SD 3.4 which, in fact, indicate a moderate BBB impairment if compared with the values reported in malignant brain tumours using the same technique. ${ }^{14}$ Furthermore, the areas with increased $\mathrm{Ki}$ values do not show a parallel increase of $\mathrm{Vp}$ values (see table 2 ), suggesting the presence of a normal vascular space in the plaques. The discrepancy with malignant brain tumours, which usually show an increase of both $\mathrm{Ki}$ and $\mathrm{Vp}$ values, could be explained by the lack of an evident vessel neoformation in multiple sclerosis plaques.

As shown in fig 1, the multiple sites of active demyelinisation may display, at the same time in the same patient, different degrees of BBB alterations.
Although pathological studies have demonstrated that only "fresh plaques" show a BBB disruption, it is still unknown whether a relationship may exist between the entity of BBB damage and the "age" of the plaque. Theoretically one may argue that the temporal evolution of BBB damage in multiple sclerosis may reflect the underlying inflammatory reaction, thus resulting in a greater BBB alteration during the "peak" of the cellular infiltration. Future sequential quantitative PET studies will be necessary in order to clarify further this issue.

In conclusion, this study demonstrates that PET using 68-Ga-EDTA is a sensitive method for the detection and measurement of BBB dysfunction associated with multiple sclerosis and may be usefully employed to evaluate the effect of therapies on BBB abnormalities during the exacerbation of the disease.

The authors are indebted to Aldo Bartiromo for his technical contribution. Mrs Patrizia Franco edited the manuscript. This work was supported by a grant from "Ministero Pubblica Istruzione, Progetti di Interesse Nazionale $40 \%$ ", and by a grant from the National Council of Research (CNR) "Progetti Strategici".

\section{References}

1 Broman T. Blood-brain barrier damage in multiple sclerosis, supravital test observations. Acta Neurol Scand 1964;40 (Suppl 10):21-4.

2 Weinstein MA, Lederman RJ, Rothner AD, Duchesneau PM, Norman D. Interval computed tomography in multiple sclerosis. Radiology 1978:129:689-94.

3 Aita JF, Bennet DR, Anderson RE, Ziter F. Cranial CT appearance of acute multiple sclerosis. Neurology 1978;5:32-9.

4 Hershey LA, Gado MH, Trotter JL. Computerized tomography in the diagnostic evaluation of multiple sclerosis. Ann Neurol 1979;5:32-9.

5 Ebers GC, Vinuela FV, Feasby T, Bass B. Multifocal CT enhancement in multiple sclerosis. Neurology 1984;34:341-6.

6 Barret L, Drayer B, Shin C. High-resolution computed tomography in multiple sclerosis. Ann Neurol 1985;17:33-8.

7 Sears ES, McCammon A, Bigelow R, Hayman LA. Maximizing the harvest of contrast enhancing lesions in multiple sclerosis. Neurology 1982;32:815-20.

8 Vinuela FV, Fox AJ, Debrun GM, Feasby TE, Ebers GC. New prospectives in computed tomography in multiple sclerosis. AJNR 1982;3:277-81

9 Brooks DJ, Beaney RP, Lammertsma AA, et al. Quantitative measurement of blood brain barrier permeability using rubidium-82 and positron emission tomography. $J$ Cereb Blood Flow Metab 1984;4:535-45.

10 Lockwood AH, Bolomey L, Napoleon F. Blood-Brain barrier to ammonia in humans. $J$ Cereb Blood Flow Metab 1984;4: 516-22.

11 Lammertsma AA, Brooks DJ, Frackowiak RSJ, Heather JD, Jones T. A method to quantitate the fractional extraction rubidium-82 across the blood-brain barrier using positron emission tomography. J Cereb Blood Flow Metab 1984;4: 523-34.

12 Hawkins RA. Phelps ME, Huang SC, et al. A kinetic evaluation of blood brain barrier permeability in human brain tumours 
with 68-Ga EDTA and positron computed tomography. $J$ Cereb Blood Flow Metab 1984;4:507-15.

13 Kessler RM, Goble JC, Bird JH, et al. Measurement of blood. brain barrier permeability with positron emission tomography and 68-Ga EDTA. J Cereh Blood Flow' Metah 1984:4:323-8.

14 Iannotti F, Fieschi C, Alfano B, et al. Simplified and non-invasive PET measurement of blood-brain barrier permeability. $J$ Comput Assist Tomogr 1987;11:390-7.

15 Schumacher GA, Beebe G, Kibler RF. Problems of experimental trials of therapy in multiple sclerosis: report by the panel on the evaluation of experimental therapy in multiple sclerosis. Ann NY Acad Sci 1965:122:552-68.

16 Gjedde A. High and low affinity transport of d-glucose from blood to brain. $J$ Neurochem 1981;36:1463-71.

17 Patlak CS, Blasberg RG, Fentermacher JD. Graphical evaluation of blood to brain transfer constants from multiple time uptake data. J Cerehr Blood Flow Metab 1983;3:1-7.

18 Patlak CS, Blasberg RG. Graphical evaluation of blood to brain barrier transfer constant from multiple time uptake data. Gen- eralizations. J Cerebr Blood Flow Metab 1985;5:584-90.

19 Ericson K, Bergstrom M, Eriksson L, et al. Positron emission tomography with 68-Ga-EDTA compared with transmission computed tomography in the evaluation of brain infarct. Acta Radiol 1981;22:385-98.

20 Ilsen HW, Sato M, Pawlik G, Herholz K, Wienhard K, Heiss WD. 68-Ga-EDTA positron tomography in the diagnosis of brain tumours. Neuroradiology 1984;26:393-8.

21 Jarden JO, Dhawan V, Kearfott KJ, Rottemberg DA. Measurement of brain/tumor capillary permeability using $82 \mathrm{Rb}$ and positron emission tomography. Ann Neurol 1984;16:131.

22 Mosskin M, Von Holst H, Ericson K, Noren G. The blood tumor barrier in intracranial tumors studied with $\mathrm{X}$-ray computed tomography and positron emission tomography using 68-GaEDTA. Neuroradiology 1986;28:259-63.

23 Schlageter NL, Carson RE, Rapoport SI. Examination of bloodbarrier permeability in dementia of Alzheimer type with $68 \mathrm{Ga}$ EDTA and positron emission tomography. J Cereb Blood Flow Metab 1987:7:1-8. 